\title{
ADVANCED INTERVENTION PLANNING FOR TRANSCATHETER AORTIC VALVE IMPLANTATIONS (TAVI) FROM CT USING VOLUMETRIC MODELS
}

\author{
Sasa Grbic ${ }^{\star \dagger}$, Razvan Ionasec ${ }^{\star}$, Tommaso Mansi ${ }^{\star}$, Bogdan Georgescu ${ }^{\star}$, \\ Fernando Vega-Higuera ${ }^{\ddagger}$,Nassir Navab ${ }^{\dagger}$, Dorin Comaniciu ${ }^{\star}$ \\ * Imaging and Computer Vision, Corporate Technology, Princeton, USA \\ $\dagger$ Computer Aided Medical Procedures, Technical University Munich, Germany \\ $\ddagger$ Siemens AG, Healthcare Sector, Forchheim, Germany
}

Aortic valve stenosis is a serious heart disease affecting a large group of elderly people. Recently minimal invasive procedures, such as the Transcatheter Aortic Valve Implantation (TAVI), are beginning to substitute conventional surgical techniques. Current methods [1] can extract basic biomarkers for TAVI such as optimal C-arm angulations, area and diameter measurements. However as the most prevalent TAVI complications (stroke and paravalvular leakages) are correlated with calcium and leaflet interactions within the valve a more advanced solution is needed. We propose a fully integrated system to extract automatically the patient specific model of the aortic valve including the volumetric model of the aortic valve leaflets and calcium from high resolution single phase CT. Based on the volumetric model advanced clinical parameters can be derived and used for e.g. patient selection, paravalvular leakage prediction and patient stroke risk assessment. We employ robust machine learning algorithms to estimate the valve model parameters. A multi-class classification method is introduced to label regions of calcium, leaflet and blood pool within the aortic valve and extract volumetric models of the aortic valve leaflets. Extensive quantitative and qualitative experiments on 198 volumetric data sets demonstrate an accurate DICE similarity score, i.e. 0.7 for the aortic valve leaflets and 0.86 for calcium tissue. Within 6 seconds a complete patient-specific model of the aortic valve can be estimated.

\section{INTRODUCTION}

Valvular heart disease (VHD) affects a large number of people and often requires costly diagnostic and interventional procedures and long-term management [2]. Traditionally, valvular heart disease has been treated with surgical repair or replacement. Over the last several years, there have been important advances in concepts, tools, techniques, and patient selection for treatment of valvular heart disease using nonsurgical procedures. One of the most prevalent procedures is the Transcatheter valve implantation (TAVI) where a replacement valve is delivered via a catheter using one of several

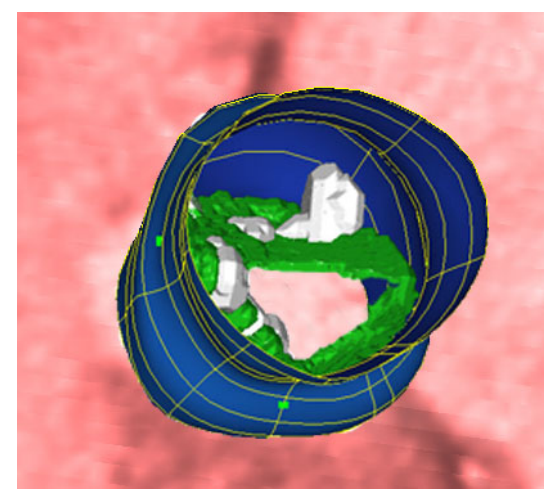

Fig. 1. Patient specific volumetric model of the aortic valve estimated from high resolution single phase CT. Model in blue represents the aortic valve root, green the aortic valve leaflets and white calified regions within the aortic valve.

access methods: transfemoral, transapical, subclavian and direct aortic. The procedure offers the potential to reduce procedural morbidity, mortality, and costs of valve treatment and is currently being utilized in non-operable and high-risk surgical patients [2].

Computed Tomography (CT) has been emerging as the main pre-operative imaging modality, which can provide key biomarkers for patient selection and procedure planning including: aortic valve annulus for device sizing [3] and orthogonal angulations for C-arm positioning [4]. Recent literature reports indicate that more complex biomarkers, such as calcium volume / distribution and leaflet tissue characteristics, can become important predictors for outcome success of TAVI procedures [5]. However current planning tools allow only for manual measurements, which are not reproducible, and enable only the extraction of basic parameters.

In the current field of medical image analysis there has been several proposals to construct models from established diagnostic modalities [6]. In the context of valvular disease management, the authors in [7] proposed to estimate mitral valve models from MRI. [1] and [8] proposed the modeling 
of the aortic valve from cardiac CT.

However, these methods were evaluated on a smaller data set where challenging diseased cases such as severe calcifications displayed in aortic valve TAVI patients were not contained. Moreover, previous methods only focus on surface models of the aortic valve anatomy, neglecting the true volumetric physiology of the aortic root and leaflets.

In this paper we propose a fully automated method to estimate a patient-specific volumetric model of the aortic valve and corresponding tissue characterization for TAVI planning. We use discriminative learning-based methods to estimate key anatomic landmarks and the shape of the aortic root and leaflets. Constrained by the previous model, a multi-class classification method is introduced to label regions of three tissue types: calcium, leaflet and blood pool. A final markov random field is constructed and optimized using graph-cuts to delineate the final volumetric model of the aortic valve. Our system was trained on a large data base containing 198 data sets with a variety of cardiovascular diseases including stenotic aortic valve cases with severe calcifications.

Our method provides a significant advantage to the current workflows as it adds capability for advanced analysis. Tissue characterization, especially calcium and its relation with native anatomies which can be useful to analyse the two most dramatic side effects of TAVI: paravalvular leakages and stroke.

\section{ESTIMATION OF AORTIC VALVE MORPHOLOGY}

We propose a physiological model of the aortic valve capable to capture complex morphological and pathological variations. The central anatomical structures consist of 11 landmarks including three commissures, three hinges, three leaflet tips, two ostias and the aortic root. To efficiently handle the anatomical complexity, the model representation and corresponding parametrization is constructed hierarchically using 1) the non-rigid landmark model $m$ and 2) the full surface model $M$ (see Fig. 2). The landmarks $m$ define key anatomical and physiological properties of the aortic valve and are modeled with 9 points: R-, $\mathrm{N}$ - and L-hinges, NL-, RN-, NLcommissures, N-, L- and R-leaflet tips. The aortic root surface is represented as a tubular grid. Discriminative learning-based methods are utilized to estimated the final model parameters. For the anatomical landmarks $m$ and aortic valve root surface $M$ estimation we employ the method proposed by [9].

\section{TISSUE CHARACTERIZATION AND VOLUMETRIC MODEL ESTIMATION}

\subsection{Multi-class tissue Classification}

We formulate our problem as a 3-class classification. Within the aortic valve root $M$ we compute custom features $x_{i}$ from each voxel $i$ (see section 3.1.1) and assign it to one of three classes: calcifications $C_{C}$, leaflet tissue $C_{L}$ and blood pool $C_{B}$. We aim to find a learning model $\mathcal{H}$ such that

$$
\mathcal{H}\left(x_{i}\right)=y_{i} \quad y_{i} \in\left\{C_{C}, C_{L}, C_{B}\right\}
$$

where $y_{i}$ is the class label for voxel $i$. We utilize binary boosting classifiers using geometric and appearance features to discriminate the voxels. In order to obtain the final class label the 1-vs-all approach is utilized [10]. Hereby the voxel $i$ is assigned to the class with the maximum probability response of the classifier $\mathcal{H}$.

\subsubsection{Geometric and Data Features}

We use data and novel geometric features to discriminate the samples. As data features we extract for each voxel $i$ its intensity in Hounsfield units $I(i)$.

$$
x_{i}(0)=I(i) \quad x_{i} \in \mathbb{R}^{10}
$$

The geometric driven features $x_{i} \in \mathbb{R}^{1 \ldots 10}$ are computed as distances to the previously estimated landmarks $m$ (see Fig. 2).

$$
x_{i}(j=1 \ldots 9)=\left\|i-m_{j}\right\| \quad x_{i} \in \mathbb{R}^{10}
$$

The intuition behind the geometric features is to capture geometric information of tissue distribution relative to the hinge, commissures and leaflet tips landmarks $m$.

\subsubsection{Training}

In order to train the binary classifiers $\mathcal{C}_{C}, \mathcal{C}_{L}$ and $\mathcal{C}_{B}$, each positive example $x_{i}$ is assigned with a class label $y_{i} \in\left\{C_{C}, C_{L}, C_{B}\right\}$ extracted from user annotations. The calcification examples are generated by an expert user defining a volume specific threshold. Only responses within the previously estimated aortic valve root model $M$ were taken. The ground-truth for the leaflet tissue position is extracted using a semi-automatic segmentation approach. The blood pool is assumed to be the remaining voxels when calcification and leaflet tissue is subtracted from the whole set of voxels within the aortic valve root $M$. Thus we train three binary classifiers: $\mathcal{C}_{C}$ (calcium), $\mathcal{C}_{L}$ (leaflets) and $\mathcal{C}_{B}$ (blood pool) using the probabilistic boosting tree [9]. The feature responses for each class $C_{C}, C_{L}$ and $C_{B}$ are shown in Fig. 2).

\subsubsection{Testing}

First landmarks $m$ and the point distribution model $M$ are estimated. All pixels inside the aortic valve are evaluated using the classifiers $\mathcal{C}_{C}, \mathcal{C}_{B}$ and $\mathcal{C}_{L}$. The final voxel $i$ is assigned to the class label with maximum probability. 


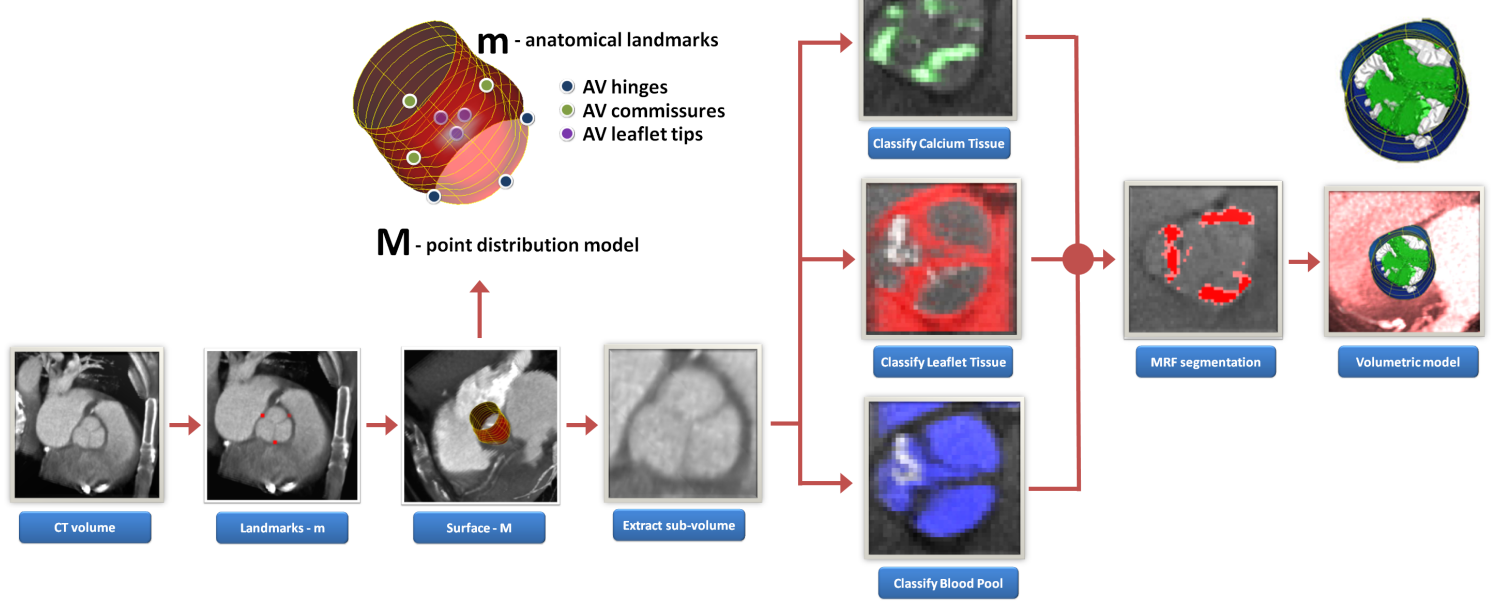

Fig. 2. Diagram showing the model estimation approach for the volumetric aortic valve model consisting of 9 anatomical landmarks $m$ ( 3 commissures, 3 hinges and 3 leaflet tips), the aortic root surface $M$ and the final volumetric aortic leaflet models.
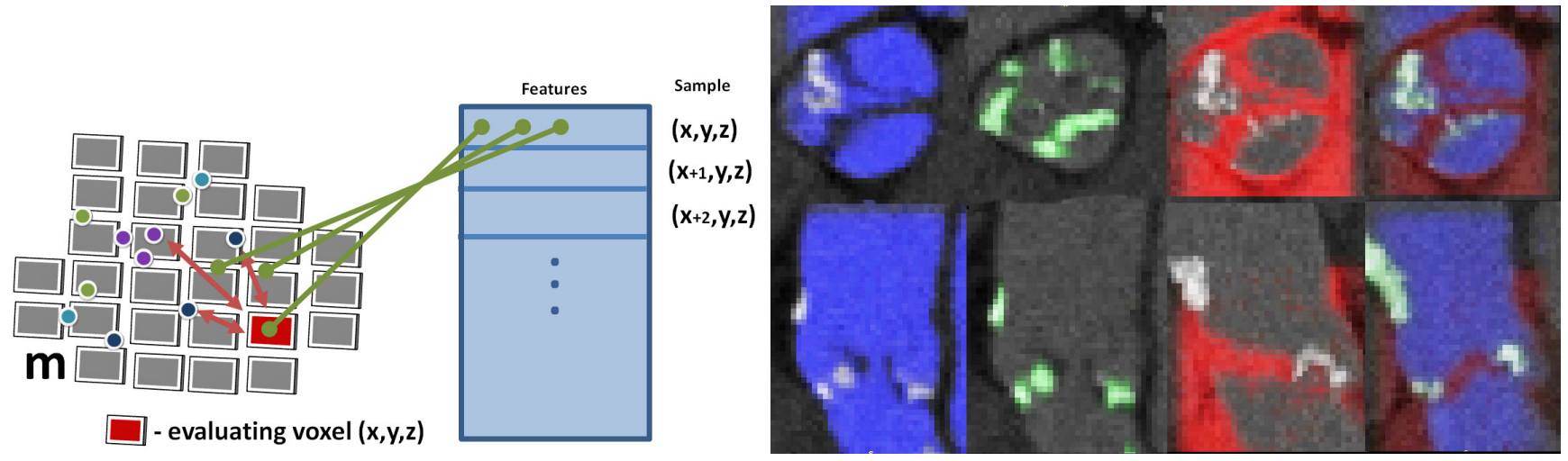

Fig. 3. Left: Custom geometric and data features utilized to classify tissues within the aortic valve root. Right: Classifier responses for different classes of tissues overlayed on the extracted subvolume. The probability map for the blood pool is overlayed on the blue channel, leaflet tissue in the red and calcium in the green color channel.

\subsection{Volumetric Leaflet tissue segmentation}

The final volumetric segmentation of the leaflet tissue is formulated as a markov random field (MRF) and optimized using graph-cuts [11]. Hereby the image segmentation problem can be viewed as a labeling process to label the voxel set $\mathrm{Q}$ by minimizing an energy function:

$$
E(L)=\sum_{p \in Q} D_{p}\left(f_{p}\right)+\sum_{q \in N(p)} V_{p, q}\left(f_{p}, f_{q}\right)
$$

where $E(L)$ is the energy, $\mathrm{p}$ and $\mathrm{q}$ are voxels, $\mathrm{N}$ is the neighborhood formed from the vertex connectivity, $D_{p}\left(f_{p}\right)$ measures the cost of assigning the label $f_{p}$ to pixel $p$, and $V_{p, q}$ measures the cost of assigning the labels $f_{p}, f_{q}$ to the adjacent pixels $p, q$. The positive and negative seeds are used from the tissue classification stage. The outside of the esti- mated aortic valve root model $M$ are set as negative seeds. Voxels classified as leaflets and calcified tissue are set as positives. The binary solutions assign the uncertain voxels either as leaflet tissue or as background. The final surface model of the leaflets is extracted from the classified voxels using the marching cube algorithm. The final volumetric model of the leaflets is shown in Fig. 3.

\section{EXPERIMENTAL RESULTS}

The accuracy of the proposed method was evaluated using 198 single-phase CT data sets. We selected only data sets with axial resolutions of less than $0.3 \mathrm{~mm} \times 0.3 \mathrm{~mm}$ and slice thickness less than $1 \mathrm{~mm}$. The ground-truth for training and testing was obtained through an incremental annotation process guided by experts, which include the manual placement 
of anatomical landmarks and delineation of the aortic valve root surface. The volumetric models of the leaflets, calcification and blood pool are segmented using a semi-automatic process. Our data set was split into 164 training and 34 test data sets. The reported numbers were performed on the test data set.

The accuracy of the hierarchical algorithm for landmark $m$ model estimation and the dense surface mesh $M$ was reported in prior work [9].

The validation of the volumetric models was done by comparing the accuracy of our multi-class tissue classification with the ground-truth annotations. Thus we computed the overlap agreement using the Dice Similarity Coefficient (DSC)

$$
D S C(A, B)=\frac{2|A \cap B|}{|A|+|B|}
$$

between the voxels inside the obtained segmentation and voxels inside the manual segmentation for the leaflet tissue (Fig 4 middle) and the calcification (Fig 4 right). A $D S C$ value of 0 indicates no overlap and a value of 1 indicates perfect agreement. We plot the Dice scores in respect to the tissue volume size in Fig. 4 for the test data.
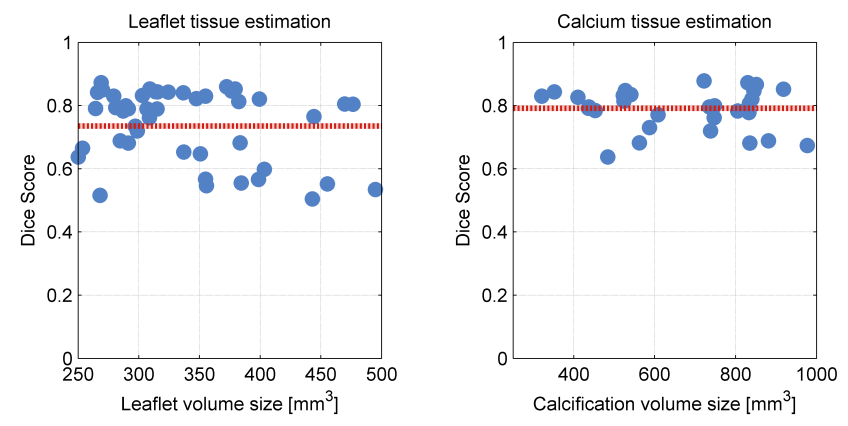

Fig. 4. Left: Dice score for the aortic valve leaflet segmentation $(D S C=0.73)$. Right: Dice score for the calcification segmentation inside the aortic valve $(D S C=0.79)$.

\section{CONCLUSION}

In this paper we presented a first step towards a complex planning tool for Transcatheter Aortic Valve procedures. Our system estimates a high-quality patient specific model of the aortic valve root and a volumetric model of the aortic valve leaflets. Robust machine learning techniques are employed to estimate the final aortic valve model parameters from high resolution single phase CT images. Advanced evaluations necessary for comprehensive planning such as calcification distribution assessment and tissue characteristics can be extracted from our volumetric models. In addition simulations of TAVI device deployment procedures can be performed using our volumetric models. Future work will concentrate to include the volumetric model of the aortic root.

\section{REFERENCES}

[1] Sasa Grbić, Razvan Ionasec, Dime Vitanovski, Voigt Ingmar, Yang Wang, Bogdan Georgescu, Nassir Navab, and Dorin Comaniciu, "Complete valvular heart apparatus model from 4D cardiac CT.," MICCAI 2010, vol. 13, no. Pt 1, pp. 218-26, 2010.

[2] Susheel K. Kodali, Mathew R. Williams, Craig R. Smith, Lars G. Svensson, John G. Webb, Raj R. Makkar, Gregory P. Fontana, Todd M. Dewey, Vinod H. Thourani, Augusto D. Pichard, Michael Fischbein, Wilson Y. Szeto, Scott Lim, Paul S. Greason, S. Chris Malaisrie, Pamela S. Douglas, Rebecca T. Hahn, Duolao Whisenant, Jodi J. Akin, William N. Anderson, and Martin B. Leon, "Two-year outcomes after transcatheter or surgical aortic-valve replacement," New England Journal of Medicine, vol. 366, no. 18, pp. 1686-1695, 2012.

[3] Choon Hwai Yap, Neelakantan Saikrishnan, Gowthami Tamilselvan, and Ajit P Yoganathan, "Experimental measurement of dynamic fluid shear stress on the aortic surface of the aortic valve leaflet.," Biomechanics and modeling in mechanobiology, Mar. 2011.

[4] Victoria Delgado, Joanne D Schuijf, and Jeroen J Bax, "Preoperative aortic valve implantation evaluation : multimodality imaging," Eurointervention, vol. 6, no. 2010, pp. 38-47, 2010.

[5] Ralf Koos, Andreas Horst Mahnken, Guido Dohmen, Kathrin Brehmer, Rolf W Günther, Rüdiger Autschbach, Nikolaus Marx, and Rainer Hoffmann, "Association of aortic valve calcification severity with the degree of aortic regurgitation after transcatheter aortic valve implantation.," International journal of cardiology, vol. 150, no. 2, pp. 142-5, July 2011.

[6] Alejandro F. Frangi, Daniel Rueckert, and James S. Duncan, "Three-dimensional cardiovascular image analysis," TMI, vol. 21, no. 9, pp. 1005-1010, 2002.

[7] Carlo A Conti, Marco Stevanella, Francesco Maffessanti, Salvatore Trunfio, Emiliano Votta, Alberto Roghi, Oberdan Parodi, Enrico G Caiani, and Alberto Redaell, "Mitral valve modelling in ischemic patients: Finite element analysis from cardiac magnetic resonance imaging," in Computing in Cardiology, 2010, pp. 1059-1062.

[8] I Waechter, R Kneser, G Korosoglou, J Peters, N H Bakker, $\mathrm{R}$ Van Der Boomen, and J Weese, "Patient specific models for planning and guidance of minimally invasive aortic valve implantation," in MICCAI, pp. 526-533. 2010.

[9] Razvan Ioan Ionasec, Ingmar Voigt, Bogdan Georgescu, Yang Wang, Helene Houle, Fernando Higuera, Nassir Navab, and Dorin Comaniciu, "Patient-specific modeling and quantification of the aortic and mitral valves from 4-D cardiac CT and TEE.," TMI, vol. 29, no. 9, pp. 1636-51, 2010.

[10] Ryan Rifkin and Aldebaro Klautau, "In defense of one-vs-all classification," Journal of Machine Learning Research, vol. 5, pp. 101-141, 2004.

[11] Y. Boykov and V. Kolmogorov, "An experimental comparison of min-cut/max-fow algorithms for energy minimization in vision," IEEE Trans. Pattern Anal. Machine Intell., vol. 26, no. 9, pp. 11241137, 2004. 